\title{
In Vivo Optical Imaging of Cellular Inflammatory Response in Granuloma Formation Using Fluorescence-Labeled Macrophages
}

\author{
Michel Eisenblätter*1, Jan Ehrchen*2-4, Georg Varga ${ }^{2}$, Cord Sunderkötter ${ }^{3,4}$, Walter Heindel ${ }^{1}$, Johannes Roth ${ }^{2,3}$, \\ Christoph Bremer ${ }^{1,3}$, and Alexander Wall ${ }^{1}$ \\ ${ }^{I}$ Department of Clinical Radiology, University Hospital of Muenster, Muenster, Germany; ${ }^{2}$ Institute for Immunology, University \\ Hospital of Muenster, Muenster, Germany; ${ }^{3}$ Interdisciplinary Center for Clinical Research (IZKF Muenster), University of Muenster, \\ Muenster, Germany; and ${ }^{4}$ Department of Dermatology, University Hospital of Muenster, Muenster, Germany
}

\begin{abstract}
Near-infrared imaging such as fluorescence reflectance imaging (FRI) and fluorescence-mediated tomography (FMT) yields high signal-to-noise ratios (SNRs) and should thus be well suited for cell-tracking studies. Extravasation of monocytes or macrophages $(\mathrm{M} \phi \mathrm{s})$ is one of the earliest events in inflammation. The purpose of this study was to assess whether FRI and FMT allow for the visualization and quantification of early inflammatory processes by tracing the migration of fluorescence-labeled murine M $\phi s$ in a cutaneous granuloma model. Methods: M $\phi s$ were labeled with a membrane-selective carbocyanine dye (1,1-dioctadecyl-3,3,3,3-tetramethylindotricarbocyanine iodide [DiR]). Cellular viability and function (nitric oxide production, phagocytosis, adherence) were assessed in vitro. Local inflammation was induced in mice by the subcutaneous injection of polyacrylamide gel pellets including or excluding a strong inflam-

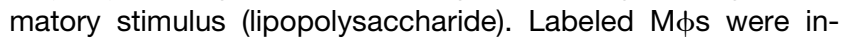
jected intravenously, and FRI and FMT were performed up to 7 d. SNRs were calculated for the pellets, and the 3-dimensional distribution of M $\phi$ s was assessed using FMT. Cells were harvested from gel pellets and analyzed by flow cytometry. Results: DiR labeling did not affect cell viability or cell function. FRI revealed the migration of labeled $M \phi s$ into gel pellets and the homing of $\mathrm{M} \phi \mathrm{s}$ to different body compartments. The lipopolysaccharide-containing pellets exhibited significantly higher SNRs than did pellets without lipopolysaccharide. FMT showed that $\mathrm{M} \phi \mathrm{s}$ distributed mainly in the periphery of the pellets. The cellular infiltrates extracted from the harvested pellets revealed the presence of approximately 10\%-23\% DiR-positive M $\phi s-$ expressing typical markers, confirming the transendothelial migration of injected M $\phi s$. Conclusion: The tagging of M $\phi s$ with DiR allows the noninvasive tracking of inflammatory cells for several days in vivo. FRI and FMT are versatile techniques to monitor and quantify cellular inflammatory responses in vivo.
\end{abstract}

Received Dec. 7, 2008; revision accepted Jun. 9, 2009.

For correspondence or reprints contact: Alexander Wall, Department of Clinical Radiology, University Hospital Muenster, Albert-SchweitzerStrasse 33, D-48129, Muenster, Germany.

E-mail: walla@uni-muenster.de

${ }^{*}$ Contributed equally to this work.

COPYRIGHT @ 2009 by the Society of Nuclear Medicine, Inc.
Key Words: optical imaging; granuloma formation; DIR; cyanine dye; fluorescence-mediated tomography

J Nucl Med 2009; 50:1676-1682

DOI: 10.2967/jnumed.108.060707

$\mathbf{O}_{\mathrm{r}}$ ptical imaging is an appealing concept for studying cell migration in vivo. Specifically, in the near-infrared range (NIR) background fluorescence is minimal, yielding excellent signal-to-noise ratios (SNRs) and thus high sensitivity of the technique for detecting even small amounts of cells. Further, fluorophores emitting in the NIR can sufficiently penetrate the tissue, even from deeper sections, and allow detection over a longer time (1). Moreover, imaging techniques for optical imaging are rapidly developing, ranging from simple surface-weighted reflection (fluorescence reflectance imaging [FRI]) to fluorescence-mediated tomography (FMT) approaches. The latter technology is capable of delivering quantitative information on 3-dimensional fluorochrome distribution in vivo.

The recruitment of immune cells and especially monocytes from the blood into tissue is crucial for the development and maintenance of inflammation (2). Interference with the mechanisms of monocyte extravasation represents an emerging new therapeutic strategy $(3,4)$. After extravasation, monocytes can differentiate into macrophages and dendritic cells. They are crucial for nearly every step of an immune reaction including the initiation of inflammation, clearance of infectious agents or tumor cells, initiation of an adaptive immune response, and resolution of inflammation $(5,6)$.

Thus, the noninvasive tracing and monitoring of monocytes or macrophages $(\mathrm{M} \phi \mathrm{s})$ in vivo by optical imaging could allow for the better localization, visualization, and 
quantification of these processes for both scientific and clinical applications.

Therefore, in this work we thought to adapt a commercially available cell-tracker dye for in vivo migration studies of murine $\mathrm{M} \phi$ s (thus enabling the imaging of early inflammatory processes). For this purpose, a new labeling protocol for rapid and safe $\mathrm{M} \phi$ s labeling was developed, and the feasibility of the project was tested in a granuloma inflammation model in mice. Imaging results were correlated to flow cytometry analysis of harvested $M \phi s$, and dose-response experiments were performed to assess the system sensitivity.

\section{MATERIALS AND METHODS}

\section{Preparation of Bone Marrow-Derived Macrophages}

$\mathrm{M} \phi \mathrm{s}$ were cultured in vitro from bone marrow progenitor cells as described previously (7).

\section{Labeling Protocol}

The commercially available lipophilic tracer 1,1-dioctadecyl3,3,3,3-tetramethylindotricarbocyanine iodide (DiR; Molecular Probes Inc.) (8) was used in all experiments. The substance has its excitation or emission spectrum in the NIR (excitation spectrum, $750 \mathrm{~nm}$; emission spectrum, $782 \mathrm{~nm}$ ) and can be solved in ethanol $(0.98 \mathrm{mmol} / \mathrm{L})$. In preliminary experiments, the labeling efficiency of DiR was determined in a phantom, using increasing concentrations of DiR (0.98-197 nmol/L).

For the following experiments, monocytes $\left(1 \times 10^{5} / \mathrm{mL}\right.$ of medium) were transfected with $2 \mu \mathrm{L}$ of DiR-labeling solution (19.7 $\mu \mathrm{mol} / \mathrm{L}$ final concentration) for $5 \mathrm{~min}$, washed 3 times with phosphate-buffered saline (PBS), and resuspended in medium. The labeling efficiency was controlled by flow cytometry. Because of some variability in the labeling intensity between different preparations of primary murine $\mathrm{M} \phi \mathrm{s}$, cells from the same labeling reaction were used for every set of in vivo experiments.

\section{Imaging Experiments}

In Vitro Microscopy. For microscopic examination of stained cells, Mфs were labeled and allowed to adhere on sterile cell plates for at least $12 \mathrm{~h}$. Microscopy was performed after replacing medium by PBS using an inverted fluorescence microscope.

FRI. The whole-body multichannel small-animal imager is a prototype reflectance imager and was already described elsewhere (9). Before in vivo experiments, the detection threshold of the imager was tested in vitro. Different numbers of labeled cells (ranging between 1,000 and 250,000 cells per phantom) were resuspended in $5 \mathrm{~mL}$ of agarosis $(1.5 \%)$ and placed in transparent plastic tubes. Image acquisition times were $1 \mathrm{~s}$ for white-light images and $3 \mathrm{~s}$ for fluorescent images.

FMT. All tomographic optical imaging studies were performed by a planar small-animal FMT system (FMT 2500; VisEn Medical Inc.) that has been described in detail elsewhere $(10,11)$. Animal scan times were in the range of 2-5 min; tomographic reconstruction times on a standard desktop PC were about 1-3 min.

Vitality and Functionality Assays. To exclude any interference of DiR labeling on functional properties of M $\phi$ s, we measured the labeling stability, apoptosis, cell adhesion, immunophagocytosis of Leishmania major parasites, and production of reactive nitricoxide (NO) species in response to classic activation with interferon (INF)- $\gamma$ and lipopolysaccharide.
Testing of Labeling Stability. To test the stability of DiR labeling, M $\phi$ s were stained with DiR and cultured with T cells (1:1 ratio) in plastic culture dishes $\left(1 \times 10^{5}\right.$ cells $\left./ \mathrm{mL}\right)$. After $2 \mathrm{~d}$ of coculture, the nonadherent $\mathrm{T}$ cells were removed and stained with a fluorescein isothiocyanate (FITC)-coupled anti-CD3 antibody (Immunotools). M $\phi$ s were detached by incubation for $30 \mathrm{~min}$ at $37^{\circ} \mathrm{C}$ in a $1: 1$ mixture of $0.5 \mathrm{mM}$ ethylenediaminetetraacetic acid and culture medium. M $\phi$ s and $\mathrm{T}$ cells were subsequently analyzed by flow cytometry.

Measurement of Cell Death. Apoptosis of DiR-stained and control M $\phi$ s was visualized after a $16-\mathrm{h}$ culture period by staining with annexin V-FITC (early marker for apoptosis) and Nicoletti assay (late marker for apoptosis) as described previously $(12,13)$.

Adhesion Assay. In contrast to other leukocytes, Mфs strongly adhere to plastic surfaces. To determine adherence, macrophages $\left(2 \times 10^{5}\right.$ cells) were seeded into untreated plastic tissue culture dishes and allowed to adhere for $30 \mathrm{~min}$. Nonadhered cells were washed away, and the remaining cells were fixed with $2 \%$ glutaraldehyde for $10 \mathrm{~min}$. After washing, cells were stained with $0.5 \%$ crystal violet in $200 \mathrm{mM}$ boric acid $(\mathrm{pH} 8.0)$ for $15 \mathrm{~min}$. Cells were washed and lysed with $10 \%$ acetic acid. The optical density was determined at $560 \mathrm{~nm}$. To check linearity of the signal-to-cell ratio and calculate cell numbers, a dilution series ranging from 0.25 to $5 \times 10^{5}$ cells was performed using unstained $\mathrm{M} \phi \mathrm{s}$ as reference. The results were corrected by subtraction of background staining of the underlying matrix (14).

Phagocytosis Assay. L. major (World Health Organization nomenclature MHOM/IL/81/FE/BNI) was cultivated in Schneider Drosophila medium as described previously (15). The phagocytosis assay was performed as described previously $(16,17)$. In short, M $\phi$ s were incubated for $4 \mathrm{~h}$ with labeled parasites, and the percentage of cells that had engulfed labeled parasites was determined using flow cytometry.

NO Release. Stimulation of M $\phi$ s with INF $\gamma$ and lipopolysaccharide results in classic M $\phi$ s activation. Production of highly active NO is one of the hallmarks of M $\phi$ s activation. NO release was measured as nitrite concentration in the medium by a microplate assay using Griess reagent as described (18).

\section{In Vivo Experiments}

Cutaneous Granuloma (CG) Model. CG formation was induced by the subcutaneous injection of $1 \mathrm{~mL}$ of polyacrylamide gel (PAG) in the flank region as previously described (19). To induce inflammation, lipopolysaccharide was added to PAG pellets $(10 \mu \mathrm{g}$ of lipopolysaccharide/mL). DiR-labeled $\mathrm{M} \phi$ s or control $\mathrm{M} \phi \mathrm{s}$ were injected intravenously $24 \mathrm{~h}$ before pellet implantation. The mice were examined daily for $7 \mathrm{~d}$ after injection using FRI and FMT. For imaging studies, mice were anesthetized by intraperitoneal administration of ketamine (125-mg dose per kilogram of body weight) and xylazine (12.5-mg dose per kilogram of body weight). All experiments were approved by the institutional animal care committee.

For signal quantification, the contrast-to-noise ratio was determined by region-of-interest analysis ( $\sim 900$ pixels) in the region of the pellet. Regions of interest of the same size were placed in nontarget tissue (over the neck) and in the background beside the mouse. The SNR was calculated as $\mathrm{SNR}=\mathrm{SI}_{\text {pellet }} / \mathrm{SD}_{\text {noise. }}$.

Analysis of Cell Infiltration of Biogel Pellets. To verify extravasation and to quantify the number of DiR-stained M $\phi$ s inside the pellets, mice were sacrificed; the biogel pellets were recovered, and infiltrated cells were separated from the gel through a $70-\mu \mathrm{m}$ 
filter in cold PBS. Cells were washed with PBS, counted, stained for surface antigen expression, and analyzed using a FACScan flow cytometer equipped with CellQuest software (Becton Dickinson). The antibodies used were as follows: FITC-conjugated F4/80, phycoerythrin-conjugated CD11b, and CD115 monoclonal antibodies purchased from Becton Dickinson.

\section{Statistical Analysis}

Data are presented as mean $\pm \mathrm{SE}$ of the mean. Differences between 2 experimental groups were statistically evaluated by Student $t$ test, and differences between multiple experimental groups were evaluated by ANOVA test with Bonferroni adjustment for multiple comparisons. A $P$ value of less than 0.05 was considered to indicate significant differences.

\section{RESULTS}

The results of the in vitro experiments are listed in Supplemental Figures 1-3 (supplemental materials are available online only at http://jnm.snmjournals.org).

\section{Labeling Protocol}

The labeling of M $\phi$ s with DiR in increasing concentrations showed a gradual increase of fluorescence up to a dose of $2.0 \mu \mathrm{L}$ of $\mathrm{DiR} / 1 \times 10^{5}$ cells (corresponding to a final concentration of $19.7 \mu \mathrm{mol} / \mathrm{L}$ ), with a slight drop of fluorescence at the higher dye concentrations, probably because of cell saturation or quenching, respectively) (preliminary studies, results not shown). On the basis of these results, the standard dose of DiR used for further experiments was set to $2.0 \mu \mathrm{L} / 1 \times 10^{5}$ cells. Supplemental Figure 1 demonstrates distribution of the dye in M $\phi$ s with incorporation of DiR in virtually all cells. In preliminary phantom experiments, as few as 10,000 cells distributed in $1 \mathrm{~mL}$ of agarose gel could be easily detected using the NIRF scanner and a 3-s acquisition time. As expected, increasing numbers of cells in the phantom resulted in a linear increase of signal intensity (preliminary studies, results not shown). To verify the stability of cell labeling, we cocultured DiR-labeled monocytes in a 1:1 ratio with $T$ cells for $2 \mathrm{~d}$. We did not detect any relevant transfer of the dye from monocytes to $\mathrm{T}$ cells (Supplemental Fig. 2).

\section{Vitality and Cell Functionality Assays}

The results of vitality and functionality assays are summarized in Supplemental Figure 3. DiR staining did not result in higher rates of apoptosis (Supplemental Fig. 3C) or reduced ability of $\mathrm{M \phi s}$ to adhere to plastic surfaces (Supplemental Fig. 3D). The phagocytosis of complement opsonized L. major parasites was not compromised in labeled M $\phi$ s, compared with control cells (Supplemental

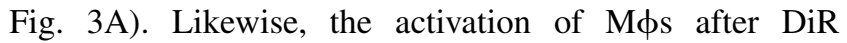
staining was not affected, as reflected by the equal NO production in response to INF $\gamma$ and lipopolysaccharide (Supplemental Fig. 3B).

\section{In Vivo Experiments}

NIRF imaging revealed the homing of labeled M $\phi$ s in different body compartments including liver, lung, and bone marrow and infiltration into gel pellets (Fig. 1). The
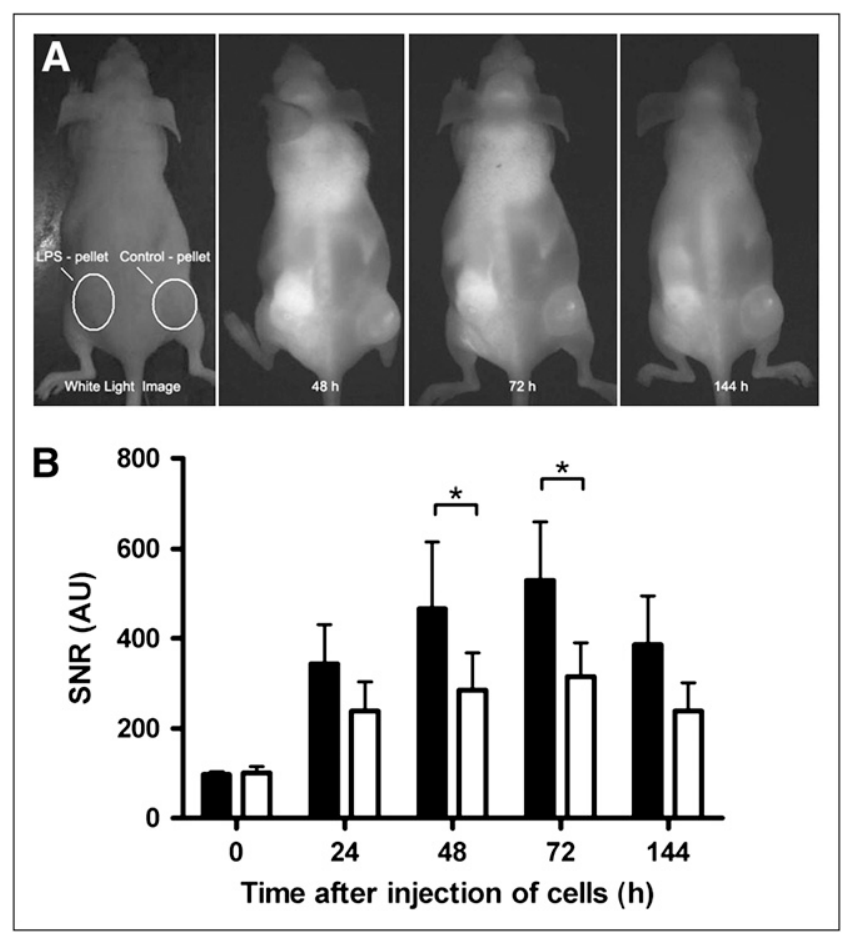

FIGURE 1. Contrast enhancement of biogel pellets after injection of labeled $\mathrm{M} \phi \mathrm{s}$. (A) Fluorescence reflectance images were repeatedly acquired up to $144 \mathrm{~h}$ after injection of labeled $\mathrm{M} \phi \mathrm{s}\left(5 \times 10^{6}\right)$. (B) Note stronger enhancement of lipopolysaccharide (LPS) pellets (right flank, घ), compared with control pellets without lipopolysaccharide (left flank, $\square$ ). Lipopolysaccharide pellets showed increasing conspicuity over first $72 \mathrm{~h}$, with slight drop of signal intensity at $144 \mathrm{~h}$. Means \pm SEMs are shown $(n=5)$. ${ }^{*} P<0.05$, Student $t$ test. $\mathrm{AU}=$ arbitrary units.

biogel pellets displayed slightly higher fluorescence signals, with SNRs up to $314 \pm 168$, than did the reference tissue (skin neck area) (Fig. 1). However, an inclusion of lipopolysaccharide in the pellets resulted in significantly higher SNRs (e.g., $72 \mathrm{~h}$ after injection: $529 \pm 292$ vs. $314 \pm 168 ; P<0.05, n=5$, Fig. 1$)$.

To visualize the spatial distribution of Mфs, FMT was performed. The tomographic scans showed that M $\phi$ s distributed mainly in the periphery of the pellets, with an approximately 2.7 times higher fluorescence signal in lipopolysaccharide-containing pellets than in controls (459 \pm 295 vs. $253 \pm 168 ; P<0.05, n=10)$ (Fig. 2). As seen in the reflectance imaging studies, the remaining cells distributed mainly to the lungs, bone marrow, and liver.

For the dose-response experiments, mice were injected with lipopolysaccharide-containing biogel pellets. The injection of increasing numbers of labeled M $\phi$ s resulted in a significantly increased FMT signal intensity. We detected an almost-linear correlation between FMT signal intensity and the number of injected cells (Fig. 3A). These data were additionally supported by counting the DiR-positive cells 


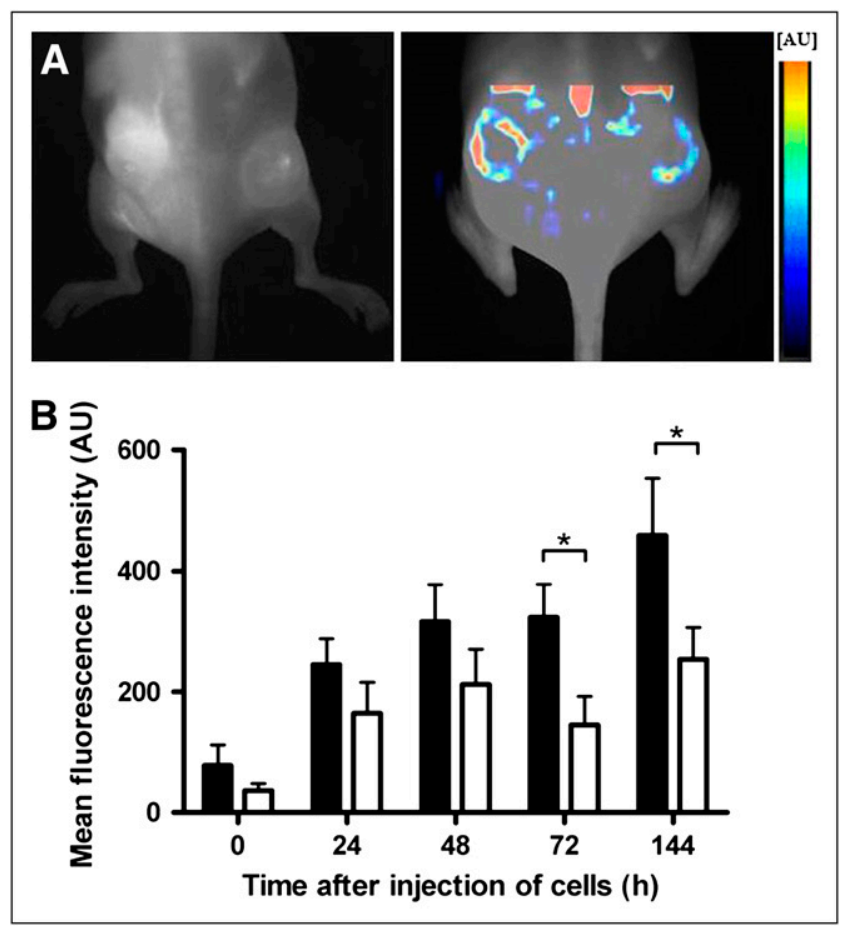

FIGURE 2. FMT of mice showed distribution of $M \phi$ s inside pellets. (A) FMT was performed $72 \mathrm{~h}$ after injection of labeled $\mathrm{M} \phi \mathrm{s}\left(5 \times 10^{6}\right)$ to resolve 3-dimensional distribution of $\mathrm{M} \phi \mathrm{s}$ in target tissue (left, NIRF image; right, color-encoded FMT). FMT confirmed FRI results and showed that $M \phi s$ distributed mainly in periphery of pellets. (B) Fluorescence quantification by FMT showed approximately 2.7 times higher signal in lipopolysaccharide-containing pellets $(\square)$ than in controls $(\square)$. Means \pm SEMs are shown $(n=10)$. ${ }^{\star} P<0.05$, Student $t$ test. $\mathrm{AU}=$ arbitrary units.

recovered from the pellets. In agreement with the FMT data, the intravenous injection of increasing numbers of DiR-stained $\mathrm{M} \phi \mathrm{s}$ resulted in increasing numbers of DiR-positive cells recovered from the pellets (Fig. 3B). DiR-positive cells recovered from the biogel pellets were uniformly positive for the macrophage differentiation marker F4/80 (Fig. 4) and the macrophage colonystimulating factor receptor (data not shown). This clearly confirms that the fluorescence signals obtained from the biogel pellets are the result of physiologic extravasation and differentiation of the intravenously injected DiRlabeled M $\phi$ s. In agreement with previously published data, we found that DiR- and F4/80-negative cells were exclusively granulocytes, as revealed by CD11b staining (marker for macrophages and granulocytes). There were no DiRpositive-F4/80-negative cells. Thus, in accordance with our in vitro data on labeling stability (Supplemental Fig. 2), there was no evidence that in vivo $\mathrm{M} \phi$ s lose their label to other cells (granulocytes) during the observation period.

Up to $23 \%$ of the cells recovered from the biogel pellets were DiR-positive $\mathrm{M} \phi \mathrm{s}\left(1 \times 10^{7}\right.$ cells injected intravenously), and up to $27 \%$ of $1 \times 10^{7}$ injected cells could be recovered from the biogel pellets. The results of flow cytometry also allowed an approximate calculation of cell numbers necessary for the visualization of inflammation; in our case, approximately $5 \times 10^{5}$ cells have to be distributed in the pellet to provide a strong signal for optical imaging (Fig. 3B).

\section{DISCUSSION}

Understanding the pathophysiology of inflammatory diseases on the cellular and molecular levels remains a major challenge. To elucidate cellular interactions after inflammatory stimuli, in vivo imaging tools-including efficient cell-labeling strategies and highly sensitive imaging techniques-are of major importance. An ideal experimental cell-labeling technique should not interfere with cell viability or cellular function and should provide a stable labeling procedure for the cells to be followed.

From a variety of imaging modalities, only few techniques such as radionuclide imaging or MRI seem to be appropriate to visualize inflammatory changes in tissue (20). Radiolabeling methods usually provide poor spatial resolution and are limited with respect to long-term followup experiments by radiation decay, whereas MRI is limited
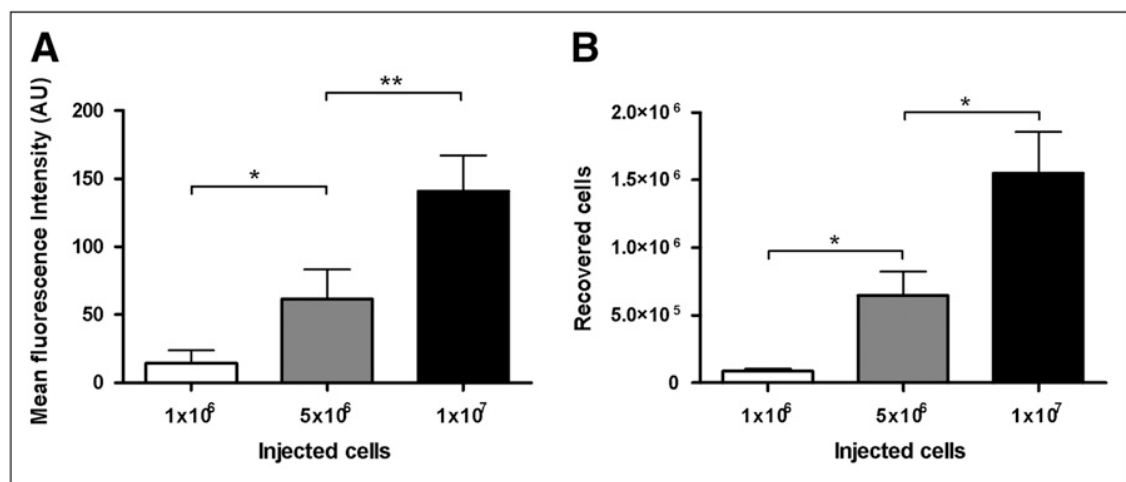

FIGURE 3. (A) Signal intensity of pellets after injection of different numbers of labeled Mфs. (B) Numbers of DiRlabeled $M \phi s$ recovered from pellets. Lipopolysaccharide-containing biogel pellets were implanted into mice, and different amounts of DiR-labeled $\mathrm{M} \phi \mathrm{s}$ were injected. FMT and cell recovery were analyzed $72 \mathrm{~h}$ after cell injection. FMT showed clear increase of signal intensity dependant on number of injected cells (A). There was also clear dependency between amount of injected cells and numbers of DiR-positive M $\phi s$ recovered from pellets (B). Means \pm SEMs are shown $(n=5)$. ${ }^{*} P<0.05$. ${ }^{\star \star} P<0.01$, ANOVA. AU $=$ arbitrary units. 
FIGURE 4. Migration of DiR-stained $M \phi s$ in vivo. Mice were injected with $5 \times$ $10^{6}$ DiR-stained M $\phi s$. Biogel pellets were recovered $2 \mathrm{~d}$ after injection of $\mathrm{M} \phi \mathrm{s}$, and intensity of DiR fluorescence and surface antigen expression were measured by flow cytometry.
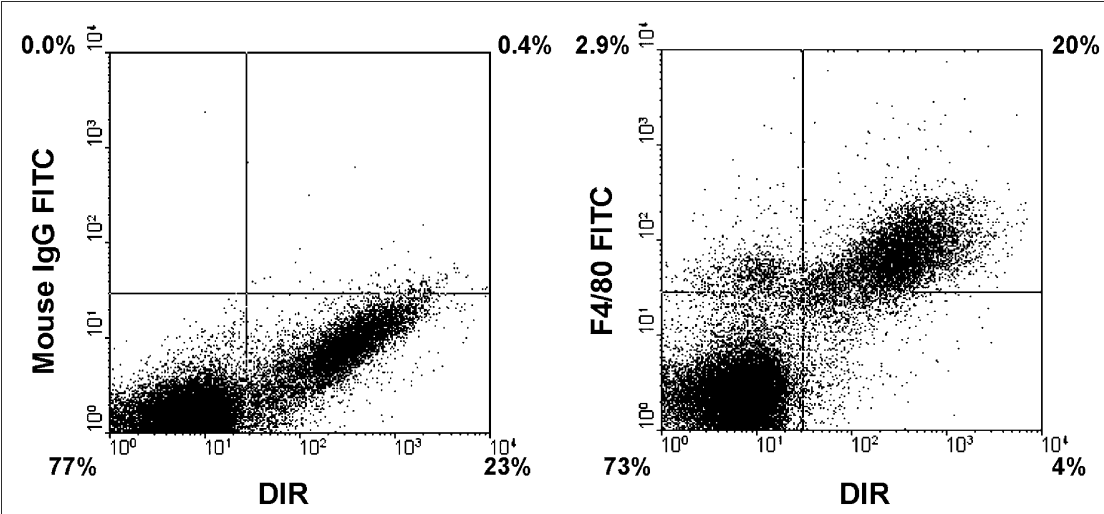

in sensitivity because of high background signal. Moreover, both techniques are cost- and time-consuming procedures and essentially lack the capability for direct ex vivo correlation. Thus, there is existing demand for new methods, ideally allowing an early detection of inflammation in tissue, the study of inflammatory process in a time course, the monitoring of inflammatory changes caused by the application of drugs or modulation of immune response, and a rapid and low-cost imaging process.

The imaging method described in this work responds to all of these criteria, providing a stable cellular label that can be detected with high sensitivity in vivo (over a long time) and correlated ex vivo (e.g., by flow cytometry analysis). We labeled $\mathrm{M} \phi \mathrm{s}$, because they represent a cell population that is crucial for basically every step of an immune reaction including the initiation of inflammation and an adaptive immune response, clearance of infectious agents or tumor cells, and resolution of inflammation $(5,6,21)$.

The labeling of M $\phi s$ with DiR proved to be a safe technique, providing flexible long-term monitoring of inflammation in tissues. The incubation of cells with only 20 $\mathrm{nmol}$ of $\mathrm{DiR} / 10^{5} \mathrm{M} \phi \mathrm{s} / \mathrm{mL}$ for $5 \mathrm{~min}$ resulted in efficient fluorochrome uptake, and no cellular toxicity was observed. An increase of the DiR dose beyond $20 \mathrm{nmol}$ of DiR/10 $\mathrm{M} \phi \mathrm{s} / \mathrm{mL}$ and an extension of the incubation time did not result in a higher fluorescence, as was probably due to saturation of the cell membranes with the dye or selfquenching of the dye, respectively.

The comprehensive tests regarding viability and functionality of DiR-labeled M $\phi$ s did not show any side effects using $20 \mathrm{nmol}$ of $\mathrm{DiR} / 10^{5} \mathrm{M} \phi \mathrm{s} / \mathrm{mL}$. However, higher doses (100 nmol of $\mathrm{DiR} / 10^{5} \mathrm{M} \phi \mathrm{s} / \mathrm{mL} ; 100 \mu \mathrm{mol} / \mathrm{L}$ ) resulted in increased $\mathrm{M} \phi \mathrm{s}$ death, indicating toxicity of the dye at this concentration.

Cell-tracker dyes were initially developed for in vitro cellmigration studies. With new imaging technology at hand, this approach can now be directly translated into in vivo imaging models. DiR has previously been used to label lymphocytes and erythrocytes and monitor the homing of these cells to different organs for up to $96 \mathrm{~h}$ after intravenous injection into mice using 2-dimensional reflectance imaging
(22). The specificity of homing was verified, and no toxicity was observed in this study. However, to the best of our knowledge there are no reports on the labeling of M $\phi$ s with NIR dyes and, more important, there are no reports on the use of cells labeled with NIR dyes for whole-body quantitative 3-dimensional imaging of inflammation in vivo.

Compared with other (alternative) tagging techniques, the labeling with DiR displays many advantages. For example, the application of fluorochrome-labeled NIRemitting antibodies directed against cell surface marker proteins has been used for the labeling of M $\phi s$ (23). However, M $\phi$ s are not a homogeneous cell population but rather encompass distinct phenotypes. For this reason, the use of cell surface markers for the detection of M $\phi$ s can be fault-prone. Besides, antibodies cannot selectively enter inflamed tissues, whereas labeled cells-if retaining their functional properties - can actively migrate to inflamed tissues and thus have a higher potential for the selective detection of inflammatory processes. Labeling of $\mathrm{M} \phi \mathrm{s}$ with fluorescent reporter genes (such as green fluorescent protein or DsRed) was also not appropriate for our project, because this technique is a laborious procedure giving relatively low yields; this procedure is also difficult to standardize. Moreover, the expression of the green fluorescent protein can influence the cellular metabolism and is therefore less suitable for the labeling of M $\phi s$ (24). Labeling with organic dyes and quantum dots still has several limitations and concerns regarding the safety of quantum dot bioconjugates for in vivo cellular imaging (25).

Reports about the use of optical tomography for celltracking studies are still rare. Recently, Nahrendorf et al. reported the successful visualization of cardiac infarction in mice using a multichannel FMT device (26). Compared with our group, the authors used an indirect labeling approach based on the intravenous injection of magnetofluorescent nanoparticles (CLIO-VT750) in mice with cardiac infarction. The M $\phi$ s and neutrophiles, which are recruited in the infarction zone, phagocytose the particles, leading to a higher concentration of fluorescent particles inside the cells. The authors described robust fluorescence in the heart region $4 \mathrm{~d}$ after infarction. A high level of correlation of the 
results of optical imaging with the results of MRI and ex vivo heart imaging was observed. However, because of the indirect labeling approach this study does not measure inflammatory cell migration in vivo. Viability studies were also not performed.

To access the potential of DiR-labeled M $\phi$ s to monitor and quantify inflammation using whole-body optical imaging in vivo, we used PAG-induced CG formation as a wellcharacterized model of inflammation (19). The model has the advantage that infiltrating cells can be recovered from the pellets after imaging and subsequently analyzed in vitro. The processes resulting in the influx of $\mathrm{M} \phi s$ into $\mathrm{CG}$ have been previously analyzed in detail. Thus, PAG induces the release of different mediators (such as complement, various lipid mediators, cytokines) on subcutaneous injection. The primary cells responsible for the initiation of $\mathrm{CG}$ formation seem to be tissue mast cells, which produce different mediators (e.g., TNF $\alpha$ ). Mice deficient in mast cells showed an almost-complete lack of cellular influx in granuloma formation $(19,27)$. The release of $\mathrm{TNF} \alpha$ from mast cells induces an influx of neutrophilic leukocytes at the early stage $(6-10 \mathrm{~h})$, which produce M $\phi$ s-recruiting chemokines, leading to recruitment of M $\phi s$ at $24 \mathrm{~h}$ and later (19). In our study, we detected M $\phi$ s infiltration into the pellets starting $24 \mathrm{~h}$ after the intravenous injection of the cells and peaking between 72 and $144 \mathrm{~h}$. The enrichment of biogel pellets with lipopolysaccharide boosted the influx of labeled macrophages, resulting in SNR values up to 2.4 times significantly higher than for the pellets without lipopolysaccharide. This finding can be easily explained by the fact that lipopolysaccharide is a strong inductor of $\mathrm{TNF} \alpha$ secretion in tissue mast cells (28).

The difference in fluorescence strength between lipopolysaccharide-containing and control CG measured by reflectance imaging and expressed in SNR was the highest $72 \mathrm{~h}$ after the injection of macrophages, with a slow decrease in the following days. The measurements conducted with optical tomography showed a slightly different result, with the highest fluorescence in lipopolysaccharide-enriched CG $144 \mathrm{~h}$ after the injection of $\mathrm{M} \phi \mathrm{s}$, which is probably due to a higher contribution of the background signal in reflectance imaging than in tomographic imaging. von Stebut et al. counted macrophages inside the biogel pellets and found the highest number $72 \mathrm{~h}$ after pellet implantation, with an approximately constant number of macrophages up to $120 \mathrm{~h}$, which is in good agreement with our FRI and FMT results (19).

In our tomographic images, we saw a higher fluorescence rim surrounding both the biogel pellet with and the biogel pellet without the addition of lipopolysaccharide, indicating infiltration of labeled M $\phi$ s into the peripheral area of CGs. This distribution resembles the microarchitecture seen in granulomatous diseases such as tuberculosis and sarcoidosis, in which macrophages are mainly located in the periphery of the granulomas.

More important, we clearly confirmed stability of M $\phi s$ labeling as well as extravasation of labeled M $\phi$ s by extracting cells from the pellet and analyzing the recovered cells by flow cytometry.

Most important, we also found a clear and linear correlation between fluorescence signals and the number of injected cells, which correlated well with the numbers of DiR-labeled cells recovered from the biogel pellets. This clearly indicates that the applied method yields quantitative results. Thus, we were able not only to localize inflammatory processes in vivo but also to quantify the extent of inflammation in vivo over 7 d. Interestingly, the results of flow cytometry analysis allow an approximate calculation of the cell number necessary for visualization of inflammation; in our case, approximately $5 \times$ $10^{5}$ cells have to be distributed in the pellet to provide a strong signal for optical imaging.

A comparison with regard to sensitivity between different imaging modalities is difficult, because different model systems and acquisition times are used. Methods reporting the tracking of a single cell with MRI are available but with an image-acquisition time of $3 \mathrm{~h}$ or longer (29). With the NIRF scanner used by our group, we could detect a cluster of as few as 10,000 labeled cells with an acquisition time of only $10 \mathrm{~s}$ (preliminary experiments, data not shown). Acquisition times up to $30 \mathrm{~min}$ are possible using new-generation NIRF scanners, which would allow a detection of even fewer labeled cells. The data on sensitivity of optical tomographic devices are not yet available, and an achieved resolution of approximately $5 \times 10^{5}$ cells distributed through the whole pellet by an acquisition time of $1 \mathrm{~min}$ in our experiments is thus only the first encouraging result.

The current study offers a solid basis for future projects. Besides its use in inflammation research, the methodology is potentially also applicable for the study of tumor biology, metastasis, stem cell homing, and more. In our case, the influence of pharmacologic agents (e.g., efalizumab and glucocorticoids) on the migration of $\mathrm{M \phi s}$ in vivo and the differential migratory behavior of pro- and antiinflammatory M $\phi s$ subpopulations are under investigation.

\section{CONCLUSION}

To our knowledge, this is the first study using the optical imaging of labeled M $\phi$ s to detect inflammation in vivo. Fluorescence cell tagging with $\mathrm{DiR}$ is an easy-to-use, inexpensive, and versatile technique, allowing rapid and safe cell labeling. The labeled cells preserve their viability and functionality. Using subcutaneous granuloma formation as an inflammation model, we demonstrated the practical applicability of the technique for the in vivo imaging of M $\phi$ s migration. The method gives both rapid and detailed spatial information about inflammatory processes in living animals and moreover offers a possibility to quantify inflammation over a time of several days in vivo. This approach offers a great potential for both experimental and diagnostic applications. Moreover, the technique offers great potential for other in vivo applications such as stem cell, tumor, or transplantation studies. 


\section{ACKNOWLEDGMENTS}

We thank Eva Nattkemper, Janine Ring, and Wiebke Gottschlich for excellent technical support. This work was supported by grants from the Interdisciplinary Center for Clinical Research (Sun2/019/07), the German Research Foundation (VO 882/2-1, FO 354/2-2, SU 195/3-1, SFB 656), the German Ministry for Research (BMBF/DLR Fkz: 01KI07100), and the European Diagnostic Molecular Imaging Network (proposal 512146).

\section{REFERENCES}

1. Weissleder R. A clearer vision for in vivo imaging. Nat Biotechnol. 2001; 19:316-317.

2. Ley K, Laudanna C, Cybulsky MI, et al. Getting to the site of inflammation: the leukocyte adhesion cascade updated. Nat Rev Immunol. 2007;7:678-689.

3. Boehncke WH, Schon MP, Girolomoni G, et al. Leukocyte extravasation as a target for anti-inflammatory therapy: which molecule to choose? Exp Dermatol. 2005; 14:70-80.

4. Kelly M, Hwang JM, Kubes P. Modulating leukocyte recruitment in inflammation. J Allergy Clin Immunol. 2007;120:3-10.

5. Mosser DM. The many faces of macrophage activation. J Leukoc Biol. 2003; 73:209-212.

6. Wells CA, Ravasi T, Hume DA. Inflammation suppressor genes: please switch out all the lights. J Leukoc Biol. 2005;78:9-13.

7. Ehrchen J, Helming L, Varga G, et al. Vitamin D receptor signaling contributes to susceptibility to infection with Leishmania major. FASEB J. 2007;21:3208-3218.

8. Haugland RP. Laboratory Manual from Molecular Probes. Carlsbad, CA: Invitrogen Corporation; 2003: Section 14.4:573.

9. Wall A, Matuszewski L, Persigehl T, et al. New experimental fluorescent reflectance imaging system with multichannel imaging capability: system performance ex and in-vivo [abstract]. Radiology. 2003;229(suppl):566.

10. Montet X, Ntziachristos V, Grimm J, et al. Tomographic fluorescence mapping of tumor targets. Cancer Res. 2005;65:6330-6336.

11. Graves EE, Yessayan D, Turner G, et al. Validation of in vivo fluorochrome concentrations measured using fluorescence molecular tomography. J Biomed Opt. 2005;10:44019.

12. Fadok VA, Voelker DR, Campbell PA, et al. Exposure of phosphatidylserine on the surface of apoptotic lymphocytes triggers specific recognition and removal by macrophages. J Immunol. 1992;148:2207-2216.

13. Nicoletti I, Migliorati G, Pagliacci MC, et al. A rapid and simple method for measuring thymocyte apoptosis by propidium iodide staining and flow cytometry. J Immunol Methods. 1991;139:271-279.
14. Kueng W, Silber E, Eppenberger U. Quantification of cells cultured on 96-well plates. Anal Biochem. 1989;182:16-19.

15. Ehrchen J, Steinmüller L, Barczyk K, et al. Glucocorticoids induce differentiation of a specifically activated, anti-inflammatory subtype of human monocytes. Blood. 2007;109:1265-1274.

16. Ehrchen J, Sindrilaru A, Grabbe S, et al. Senescent BALB/c mice are able to develop resistance to Leishmania major infection. Infect Immun. 2004;72:51065114.

17. Schonlau F, Scharffetter-Kochanek K, Grabbe S, et al. In experimental leishmaniasis deficiency of CD18 results in parasite dissemination associated with altered macrophage functions and incomplete Th1 cell response. Eur J Immunol. 2000;30:2729-2740.

18. Ding AH, Nathan CF, Stuehr DJ. Release of reactive nitrogen intermediates and reactive oxygen intermediates from mouse peritoneal macrophages: comparison of activating cytokines and evidence for independent production. $J$ Immunol. 1988;141:2407-2412.

19. von Stebut E, Metz M, Milon G, et al. Early macrophage influx to sites of cutaneous granuloma formation is dependent on MIP- $1 \alpha / \beta$ released from neutrophils recruited by mast cell-derived TNF $\alpha$. Blood. 2003;101:210215.

20. Boerman OC, Rennen H, Oyen WJ, et al. Radiopharmaceuticals to image infection and inflammation. Semin Nucl Med. 2001;31:286-295.

21. Gordon S. Alternative activation of macrophages. Nat Rev Immunol. 2003;3: 23-35.

22. Kalchenko V, Shivtiel S, Malina V, et al. Use of lipophilic near-infrared dye in whole-body optical imaging of hematopoietic cell homing. J Biomed Opt. 2006; 11:050507.

23. Hansch A, Frey O, Sauner D, et al. In vivo imaging of experimental arthritis with near-infrared fluorescence. Arthritis Rheum. 2004;50:961-967.

24. Hanazono Y, Yu JM, Dunbar CE, et al. Green fluorescent protein retroviral vectors: low titer and high recombination frequency suggest a selective disadvantage. Hum Gene Ther. 1997;8:1313-1319.

25. Ohnishi S, Lomnes SJ, Laurence RG, et al. Organic alternatives to quantum dots for intraoperative near-infrared fluorescent sentinel lymph node mapping. Mol Imaging. 2005;4:172-181.

26. Nahrendorf M, Sosnovik DE, Waterman P, et al. Dual channel optical tomographic imaging of leukocyte recruitment and protease activity in the healing myocardial infarct. Circ Res. 2007;100:1218-1225.

27. Harris RR, Wilcox D, Bell RL, et al. The role of tissue mast cells in polyacrylamide gel-induced inflammation in mice. Inflamm Res. 1998;47:104108.

28. Chiba N, Masuda A, Yoshikai Y, et al. Ceramide inhibits LPS-induced production of IL-5, IL-10, and IL-13 from mast cells. J Cell Physiol. 2007;213: 126-136.

29. Dodd SJ, Williams M, Suhan JP, et al. Detection of single mammalian cells by high-resolution magnetic resonance imaging. Biophys J. 1999;76:103109. 